\title{
De la casa de locos a la asistencia especializada en hospitales. El caso de Mérida (Badajoz)
}

\section{From the madhouse to the assistance specialized in hospitals. The case of Merida (Badajoz)}

\section{Da casa de louco ao cuidado especializado em hospitais. 0 caso de Merida (Badajoz)}

\author{
Jesús Prieto Oreja \\ Enfermero Especialista de Salud Mental. Doctor por la UEX. \\ Cómo citar este artículo en edición digital: Prieto Oreja, J. (2018) De la casa de locos a la asistencia \\ especializada en hospitales. El caso de Mérida (Badajoz). Cultura de los Cuidados (Edición digital), 22(51). \\ Recuperado de <http://dx.doi.org/10.14198/cuid.2018.51.09> \\ Correspondencia: Unidad de Salud Mental. Hospital de Mérida. C/Antonio Campos Hoyos, 26. C.P. \\ 06800. Mérida (Badajoz). \\ Correo electrónico: cositoxii@yahoo.es \\ Recibido: 23/11/2017; Aceptado: 18/03/2018.
}

\section{ABSTRACT}

Objective: To know how has been the historical evolution of psychiatric care to people with mental pathology in the city of Mérida (Badajoz).

Method:This article gives a brief description of the historical development that has led to the attention to people with mental pathology from closed and total hospitalization centers to the specialized care in hospitalization units in general hospitals focusing on the city of Mérida ( Badajoz) by reviewing bibliographical sources related to the topic. Development of the theme: The journey from life-long internments in psychiatric hospitals to general and community hospitals has been long, with non-compliance with deadlines, with comings and goings, and with unfinished roads. Legislation that has influenced the future of thousands of people. Rules that have been dictated by some and fulfilled by others. The modification of the form of assistance to people with mental pathology has been temporarily marked by an attempt to open up to society
\end{abstract}

those institutions of neglect and forgetfulness that were psychiatric and that Michael Foucault at the time called heterotopias.

Conclusions:Compliance with laws and regulations is still incomplete, as enforcement is still used as a means of social control and above all behavioral control. The social norm imposed on the norm of behavior.

Keywords: Mental Health , hospital, historical development.

\section{RESUMEN}

Objetivo: Conocer cómo ha sido la evolución histórica de la atención psiquiátrica a las personas con patología mental en la ciudad de Mérida (Badajoz)

Método: En este artículo se hace una breve descripción del desarrollo histórico que ha llevado a la atención a las personas con patología mental desde centros de internamiento cerrados y totalizadores hasta la atención especializada en unidades de hospitalización en hospitales generales centrándome en la ciudad 
de Mérida (Badajoz) mediante la revisión de fuentes bibliográficas referidas al tema.

Desarrollo del tema: El camino recorrido desde los internamientos de por vida en hospitales psiquiátricos hasta la atención en hospitales generales y comunitaria ha sido largo, con incumplimiento de plazos de tiempos, con idas $\mathrm{y}$ venidas, $\mathrm{y}$ con caminos inacabados. Legislaciones que han influido en el devenir de miles de personas. Normas que han sido dictadas por unos y cumplidas por otros. La modificación de la forma de asistencial a las personas con patología mental ha ido temporalmente marcada por un intento de apertura a la sociedad de esas instituciones de abandono y olvido que eran los psiquiátricos y que Michael Foucault denominó en su momento heterotopias. Conclusiones:El cumplimiento de las leyes y normas aún sigue siendo incompleta puesto que aún se sigue recurriendo al encierro como medio de control social y sobre todo de control conductual. Lo norma social impuesta sobre la norma del comportamiento.

Palabras clave: Salud Mental, hospital, evolución histórica.

\section{RESUMO}

Objetivo: Saber o que tem sido a evolução histórica da assistência psiquiátrica para pessoas com doença mental na cidade de Mérida

(Badajoz)

Método: Este artigo fornece uma breve descrição do desenvolvimento histórico que trouxe atenção às pessoas com doença mental dos centros fechados e totes internamento de cuidados especializados em unidades de internação em hospitais gerais com foco na cidade de Mérida é ( Badajoz), revendo fontes bibliográficas referiu-se ao assunto.

Desenvolvimento do tema: A viagem das internações para a vida em hospitais psiquiátricos aos cuidados em hospitais gerais e da comunidade tem sido prazos longos, tempo perdido, com idas e vindas, e estradas inacabadas. Leis que influenciaram a evolução de milhares de pessoas. Normas que tenham sido emitidas por alguns e cumprido por outro. Alterar a forma de cuidados para as pessoas com doença mental foi temporariamente marcada por uma tentativa de abrir a sociedade das instituições que foram abandonados e esquecidos psiquiátrica e Michael Foucault chamados heterotopias em seu tempo.

Conclusões:Conformidade com leis e regulamentos ainda está incompleta, uma vez que continua a usar o fechamento como um meio de controle social e controle especialmente comportamental. regra social imposta ao padrão de comportamento.

Palavras chave: Saúde mental, hospital, evolução histórica.

\section{INTRODUCCIÓN}

El objetivo de este artículo es conocer cómo ha sido la evolución histórica de la atención psiquiátrica a las personas con patología mental en la ciudad de Mérida (Badajoz). Este conocimiento centrado en la asistencia psiquiátrica en Mérida nos servirá para poder hacer extensivo el mismo a la totalidad de la comunidad autónoma de Extremadura e incluso a otra comunidades autónomas por las similitudes en el proceso. Conocer la historia de esta asistencia no ayudará a conocer además como ha sido la legislación al respecto, como ha sido la mirada recelosa que siempre ha tenido la sociedad hacia estos pacientes y de tal vez comprobar que situaciones asistenciales que creíamos del pasado se siguen produciendo en la actualidad.

\section{MÉTODO}

En este artículo se hace una breve descripción del desarrollo histórico que ha llevado a la atención a las personas con patología mental desde centros de internamiento cerrados y totalizadores hasta la atención especializada en unidades de hospitalización en hospitales generales centrándome en la ciudad de Mérida (Badajoz) mediante la revisión de fuentes bibliográficas referidas al tema. La fuentes bibliográficas consultadas han sido sobre todo textos legales tanto de ámbito provincial, autonómico y nacional, además se han consultado textos históricos locales sobre lugares de internamiento en Mérida, y por últimos textos fundamentales sobre cómo ha sido la asistencia asilar de los paciente con patología mental. 


\section{DESARROLLO DEL TEMA}

El estudio sobre los lugares de internamiento de personas siempre tuvo un cierto halo de misterio y exotismo. El lugares "diferentes" eran el foco de la miradas curiosas de algunos investigadores, y así en el caso de sociólogos y antropólogos que dándose cuenta que el estudio de culturas externas era un tema bastante desarrollado pensaron que era hora de hacer un mirad interior dentro de la propia comunidad. Era hora de mirar las "extrañezas" propias, a así destacan los estudios de Foucault y Goffman. Era hora de hacer un repaso histórico de lo que había sucedido y hasta dónde se había llegado como demuestran autores como González de Cháves y Curado García. Era hora de saber cómo la ley y la justicia había acompañado, o no, a estos "desviados sociales" en muchos casos por obligación, como exponen autores como Degado Bueno. Es hora de saber casos puntuales como el de Mérida, que es cuerpo de estudio de este artículo.

La palabra manicomio deriva del término nosocomio, nosos (enfermedad) $\mathrm{y}$ Komáo (cuidar). Era el lugar donde se atendían a las personas enfermas, con la sustitución del término nosos por el de manía. Pero realmente,
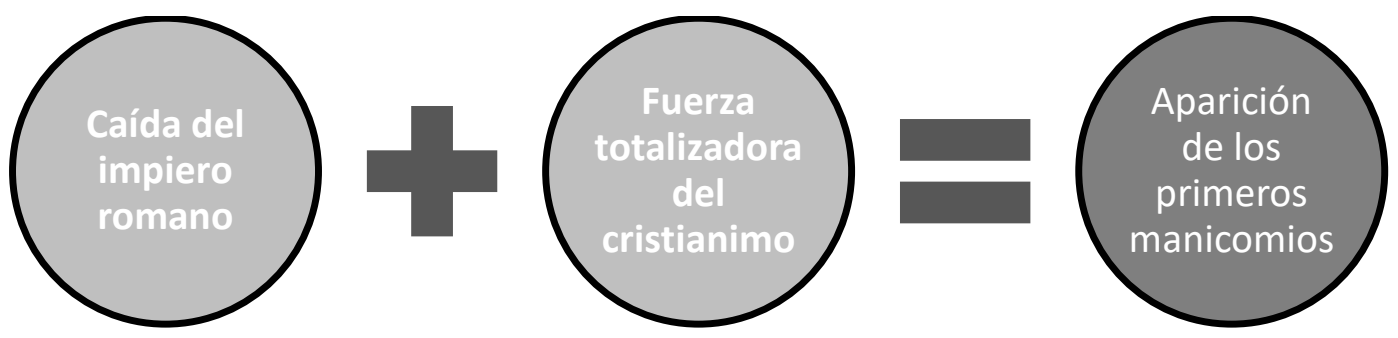

Figura 1. Condiciones históricas que llevaron a la creación de los primeros manicomios.

La relación con el paciente mental durante los siglos posteriores a la Edad Media y hasta el origen de la psiquiatría moderna fue la reclusión de los enfermos mentales en el domicilio y a cargo de la propia familia. La acción de la comunidad llegaba cuando la familia era incapaz de contener al paciente en el domicilio, y sus conductas eran violentas o de carácter delictivo. Se les encerraba entonces en celdas próximas a la Iglesia, dentro de los y aunque la voz manicomio tenga origen en terminología griega, no fueron los griegos los primeros en crear los centros de encierro para personas con trastornos mentales; es más, en estas personas se veía cierto grado de deidad y de capacidad de contacto con los dioses. Ni tampoco los romanos, sino que con la imposición de las creencias religiosas (al menos en Europa) se forjó la idea del cuidado del paciente mental en instituciones, muchas de ellas de carácter religioso. Así, mientras la cultura griega había desarrollado confianza en la individualidad y autonomía del ser humano con el culto a la razón, la cultura romana encontraba su seguridad en el estado, la organización y la ley. Con la caída del Imperio Romano se produjo el resquebrajamiento de una sociedad que presentaba cierta coherencia formada por pequeñas naciones y grupos de guerreros, que sólo podía ser contrarrestado por la única fuerza con poder totalizador: el Cristianismo (Kroll, 1973). Se encierra al desviado de Dios y no al desviado de la razón. Así, se encierra a depravados, a los padres disipadores, a los hijos pródigos, a los blasfemos, a los hombres que "tratan de deshacerse" y a los libertinos (Foucault, 1997). Figura 1. 
legislación creada por las actuaciones de estos dos médicos sirvió en Francia desde 1838 hasta 1990. El tratamiento moral fue la terapéutica "científica" que exigía y justificaba la construcción de manicomios, y consolidó socialmente esta red de instituciones específicas para la locura, obligó a reformar toda la legislación, se creó la especialidad en psiquiatría y surgieron las primeras asociaciones alienistas o superintendentes de asilos (González, 1980). Todas estas modificaciones venían impulsadas por el optimismo revolucionario francés, y aunque las condiciones de los pacientes mejoraron (al menos en Francia) éstos seguían estando encerrados, aislados y alienados. Esta medida inicialmente positiva, en cuánto intencionalidad moral, fue aprovechada por el empuje capitalista para lograr parte de sus objetivos. Y es que con el nacimiento de la Revolución Industrial se produce una problemática social en la que se le exige al individuo ser normativo, convertirse en un disciplinado social y en una mano de obra funcional. Como es lógico intuir, el paciente mental deja también de tener hueco en esa sociedad industrializada, ya que en muchos casos le resulta imposible cumplir con lo exigido. Así como señala Desviat (1990), el tratamiento moral se inserta en los esfuerzos normalizadores, típicos de la burguesía posrevolucionaria que, mediante una aparente filantropía, desarrolla una red asistencial para la población indigente buscando un cierto orden y la interiorización de unos códigos sociales, reglas de comportamiento, civilización de las pulsiones, que no es otra cosa que la alienación política y económica, condición necesaria en el orden capitalista .Se pasa del problema moral, considerado por la Iglesia, al problema de orden social considerado por el estado.

En España el internamiento en psiquiátricos de forma legalizada comienza en la Ley de la Beneficencia de 1822. Se ocupaba por primera vez de los derechos y deberes de los alienados. Más de un siglo después se publica el Decreto 105 de 3 de julio de 1931 (con modificaciones introducidas por el Decreto 27 de mayo 1932 y la Orden Ministerial de 30 de diciembre de 1932), que intenta tener una visión más progresista que la legislación anterior pero debido a las deficiencias de la infraestructura sanitaria lo convierten en inadecuado y se encuentra con muchas críticas por la forma aleatoria de aplicar tratamientos o simplemente por las formas de ingreso de las personas. Con posterioridad se crean nuevas leyes con la intención de corregir fallos y dar una mejor atención a los pacientes desde el punto de vista legal. Estas leyes serán la Ley de Sanidad Nacional de 25 de noviembre de 1944, la Ley de Hospitales de 21 de julio de 1967 , el Decreto de 24 de mayo de 1943, sobre Dispensarios de Higiene mental, la Ley de Vagos y Maleantes de 4 de agosto de 1933, sustituida por la Ley de Peligrosidad y Rehabilitación Social de 4 de agosto de 1970; posteriormente modificada por la Ley 77/1978 de 28 de noviembre (Delgado Bueno, González de Rivera, Revuelta, 1988). Figura 2.

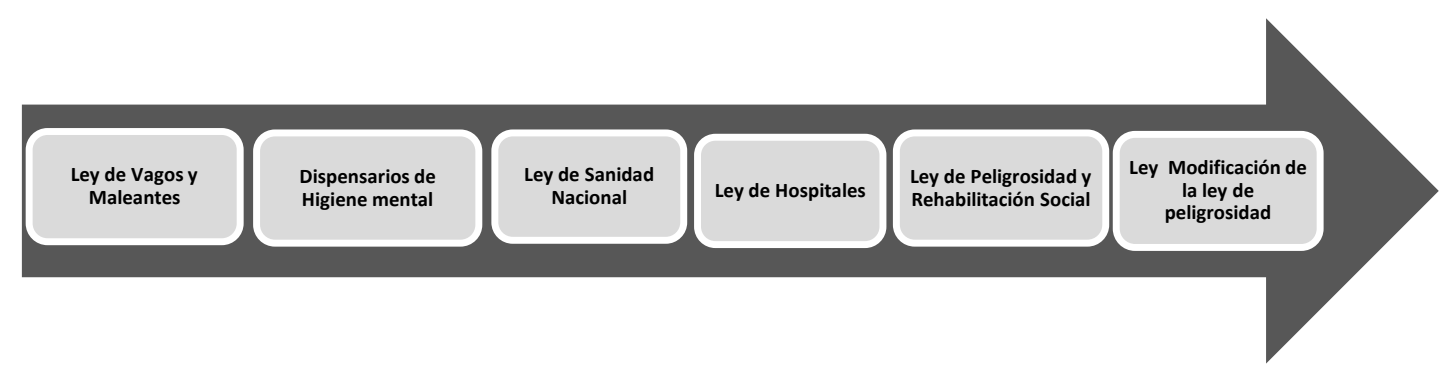

\section{$1933 \longrightarrow 1978$}

Figura 2. Principales leyes vinculadas a la salud mental de la población en el siglo XX. 
Toda la legislación anterior no daba una respuesta adecuada a los pacientes con patologías mentales a los cuales privaba de uno de sus derechos fundamentales: la libertad. La legislación española continuaba viendo al enfermo mental como alguien peligroso, y su equiparación con animales feroces o dañinos a la hora de proceder a su custodia (Bercovitz, 1980). Esta situación forzaría la necesidad de legislar nuevas leyes que cumplieran unas mínimas condiciones que dignificaran la situación de los considerados como pacientes con patología mental.

En 1978 con la entrada en vigor de la Constitución Española el ordenamiento jurídico también va a sufrir grandes modificaciones legislativas y entre ellas leyes que afectarían directamente a los personas con patología mental. Destacan dos, la primera sería la Ley Orgánica 8/83 de 25 de junio en la que se dicta que serán jueces y tribunales los que en caso de internamiento éste será según las circunstancias individuales de cada sujeto siendo la finalidad última del internamiento su recuperación y rehabilitación. La segunda, Ley de 13/1983 de 24 de octubre reforma el Código Civil en materia de tutela poniendo en vigor el artículo 211 sobre los internamientos involuntarios (que posteriormente fue sustituido por el artículo 763), y en materia de incapacitación de sujetos. La legislación anterior se redacta en papel pero la puesta en práctica de lo que imponen no se hace efectiva totalmente, lo que conlleva a la continuidad de las estructuras existentes, como son los hospitales psiquiátricos, si bien se empiezan a plantear la creación de nuevas estructuras que se adapten a nuevas situaciones socializadoras.

En el caso de Mérida (Extremadura), el primer lugar de encierro con características exclusivamente psiquiátricas fue la creación de la casa hospitalaria de dementes que abriría sus puertas el 1 de febrero de 1843. El lugar donde se abrió fue en el antiguo hospital-convento de Jesús, zona muy céntrica de la ciudad, lo cual llama la atención por crear un centro de internamiento de pacientes mentales en esa ubicación, ya que no era lo normal. El Boletín Oficial de la provincia de Badajoz del día 23 de enero de 1843 establece una casa hospitalaria de dementes para toda la provincia; en la que según reza en dicho boletín:" tendrán admisión los locos de ambos sexos de la provincia según los recursos y disponibilidad que la misma goce". El boletín tiene una interesantísima lectura. En él aparecen, desmembrando el boletín, una serie de artículos sobre las formas de actuar que en la actualidad resultan de lo más hilarante. Cabe destacar indicaciones como las mencionadas en el artículo 4 en el que se cita: " El continuado encierro, la excesiva severidad en el trato, los golpes, grillo y cadenas, jamás se usarán sin consulta del facultativo". O como en el indicado en el artículo 7 en el que se dice, haciendo referencia a las características de los cuidadores, que: " El cuidado de los dementes de uno y otros sexo estará a cargo de dos o más personas del suyo respectivo, que a honradez y providad conocida reúnan la sensatez y humanidad indispensables y que bajo la inspección de la jaula cuiden del aseo, ventilación y limpieza de las jaulas, prestando a estos infelices cuantos socorros estén en su mano; pero con la condición en todo tiempo de responder de la seguridad de los locos, y de que entre ellos reine la mayor armonía". Incluso otro en el que se hace mención específica a la diferencias de clases sociales: "Si entre los comprendidos en el artículo anterior hubiese alguno que por su clase o categoría quieran sus parientes distinguirlo sobre los demás, se le designará una habitación separada para su mayor decencia y comodidad...". O por último, y para no alargar este apartado, en el que se hace referencia a la dieta a seguir por los pacientes: "La ración por cada demente consistirá en libra y media de pan, dos onzas de garbanzos secos, una onza de tocino, cuatro de carne y una de arroz alternado con otras clases de menestras que se distribuirá en esta forma: tercera parte de pan en sopa por la mañana con caldo de cocido; la otra tercera parte con el cocido y la otra tercera parte con el caldo que se separará del mismo cocido y tocino."

El funcionamiento de dicho centro sería desde 1843 hasta 1851. En el año de la fecha de cierre se publica en el Boletín Oficial de la Provincia, el 7 de abril de 1851, que: "Debiendo verificarse la obra de reparación en el Exconvento de Descalzos de la ciudad de Mérida, para que se trasladen al mismo los dementes que se hallan en el asilo de dicha 
ciudad...”. En 1929 se realizan unas reformas que mejoran los servicios pero no la filosofía de la asistencia. Los locos seguirán, por mucho tiempo, amarrados a sus cadenas, esperando la reforma que todavía no llega (Curado, 2003).

Estando en manos de las Diputaciones Provinciales la atención a los enfermos mentales en cuanto a internamiento prolongado se refiere, y siguiendo la política de construcción de grandes hospitales, se hicieron dos en Extremadura: el de Plasencia y el de Mérida. En el caso de este último, se construyó a las afueras de la ciudad, más concretamente en la carretera que iba a Valverde de Mérida. Es en el año 1975 cuando el hospital abre sus puertas con una capacidad de 1000 camas, y llamándose: "Hospital Psiquiátrico Adolfo Díaz Ambrona”. Era y sigue siendo una estructura de características totalizadoras (Goffman, 1998).

Figura 3

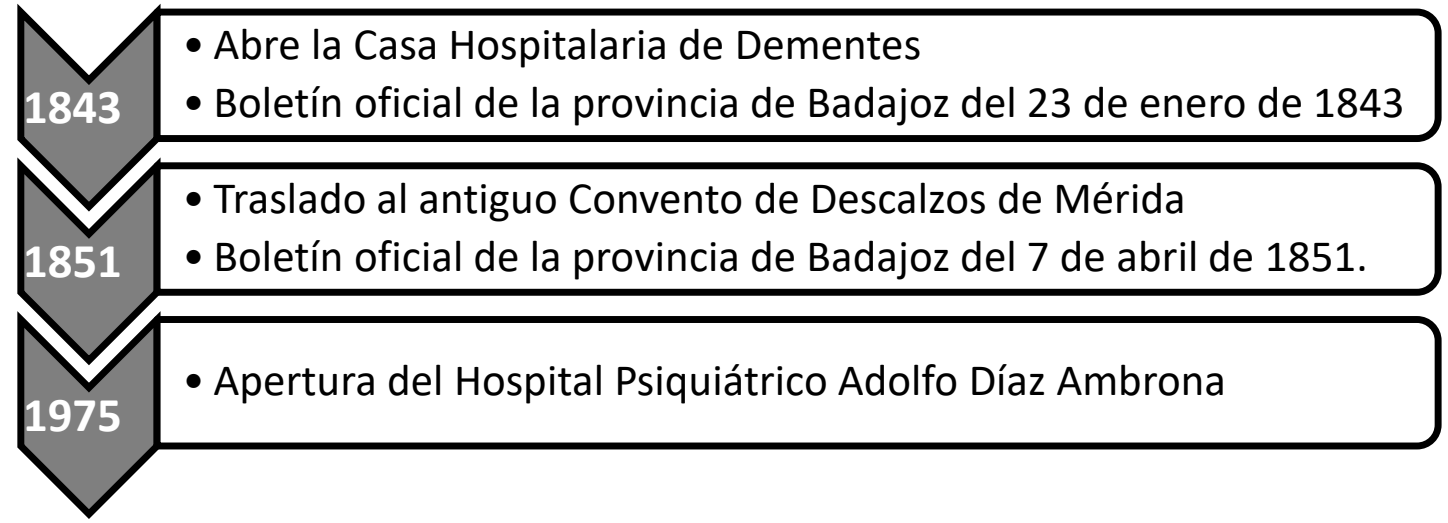

Figura 3. Cronología de aperturas de centros de internamiento psiquiátrico en Mérida.

A pesar del aislamiento geográfico y social sufrido por estos hospitales psiquiátricos, ambos serán afectados por la legislación que poco a poco se empieza a retomar en cuanto a la Salud Mental se refiere. Así en la Ley 14/1986, de 25 de abril, General de Sanidad, en el Capítulo III, artículo 20 determina la plena integración de las actuaciones relativas a la salud mental en el sistema nacional de salud y la total equiparación del enfermo mental a las demás personas que requieran servicios sanitarios y sociales. Para ello las Administraciones Sanitarias competentes deben adecuar su actuación a los principios enunciados en el mismo. En esta ley cabe destacar el punto 3 del artículo 20 en el que se indica: “La hospitalización de los pacientes por procesos que así lo requieran se realizarán en las unidades psiquiátricas de los hospitales generales”. Esta indicación legal es muy interesante ya que mientras que Cáceres y Badajoz sí cumplían la ley al tener unidades de hospitalización de psiquiatría en sus hospitales de Virgen de la Montaña (Cáceres) y Hospital Infanta Cristina (Badajoz) pertenecientes al antiguo INSALUD, no era el caso de Mérida ni Plasencia. Ambas localidades no sólo tenían centros de internamiento de largo plazo sino que además tenían las unidades de internamiento breve que según la ley deberían estar ubicadas en hospitales generales. Esta apertura en hospitales generales de las unidades de Mérida y Plasencia no se producirá hasta el 1 de febrero de 2011. Veinticinco años después de los que la ley indicaba.

Destaca el Decreto 92/2006 de 16 de mayo por el que se establece la organización y funcionamiento de la atención a la salud mental en la Comunidad Autónoma de Extremadura. En él también se hace referencia en el Art. 8, punto 5 que "Las Unidades de Hospitalización Breve estarán ubicadas e integradas en el Hospital, adaptando su estructura, recursos y funcionamiento a las características de su especialización.” Cualquiera que vea el artículo puede pensar que está claramente definido donde debe estar una unidad de agudo, pero como la ley es interpretativa, al decir Hospital no queda claro si hospital general u hospital psiquiátrico. Tocaba de nuevo esperar.

Tal vez, y con la puesta en marcha de la Ley 1/2008, de 22 de mayo, de creación de Entidades Públicas de la Junta de Extremadura, 
en la que se crea el Servicio Extremeño de Promoción de la Autonomía y Atención a la Dependencia, y con la decisión de llamar a los antiguos manicomios, que en una segunda fase pasaron a llamarse hospitales psiquiátricos, centros sociosanitarios no sólo se seguía incumpliendo la Ley General de Sanidad sino también el Decreto 92/2006. Tal vez por este motivo, o por la presión de las asociaciones de los pacientes mentales, por fin se abrieron las unidades de salud mental para patología aguda en los hospitales generales del Servicio Extremeño de Salud de Plasencia y Mérida.

Se separaban de esta forma los pacientes agudos de los hospitales psiquiátricos, que aunque en el papel pasaron a llamarse centros socio-sanitarios continuaron con las mismas características manicomiales con levísimos retoques asistenciales.

Se cerraba, al menos de momento, el recorrido de la salud mental desde los cuidados domiciliarios pasando por las órdenes religiosas llegando a los manicomios para terminar en una atención más “comunitaria”. Esperemos que este no sea el fin.

\section{CONCLUSIONES}

El cumplimiento de las leyes y normas aún sigue siendo incompleta puesto que aún se sigue recurriendo al encierro como medio de control social y sobre todo de control conductual. Lo norma social impuesta sobre la norma del comportamiento. Falta aún muchos recursos para una adecuada asistencia a los pacientes con patología mental, los cuales, a pesar de los esfuerzos de la administración, siguen siendo tratados con cierto recelo por la sociedad la cual mantiene un cierto nivel estigmatizador.

\section{FUENTES NORMATIVAS}

Ley 13/1983, de 24 de octubre, de Reforma del Código Civil en materia de tutela ley 14/1986, de 25 de abril, General de Sanidad, en el Capítulo III, artículo 2

Ley 37/1962, de 21 de julio, sobre Hospitales. Ley 77/1978, de 26 de diciembre, de modificación de la Ley de Peligrosidad Social y de su reglamento.
Ley de Bases de la Sanidad Nacional, de 25 de noviembre de 1944. Ley de Peligrosidad y Rehabilitación Social de 4 de agosto de 1970. Ley 1/2008, de 22 de mayo, de creación de Entidades Públicas de la Junta de Extremadura.

Ley de Vagos y Maleantes de 4 de agosto de 1933.

Ley Orgánica 8/1983, de 25 de junio, de Reforma Urgente y Parcial del Código Penal

\section{BIBLIOGRAFÍA}

Bercovitz Rodríguez-Cano, R. (1980). Psiquiatría y Derecho. En González de Rivera J.L., VELA, A., Arana, J. Manual de Psiquiatría. (Pp1239-1255). Madrid : Ed. Karpos.

Curado García, B.(2003). Hospitales en Mérida. Revista de estudios extremeños, 59( 1), 427-445.

Decreto 92/2006 de 16 de mayo por el que se establece la organización y funcionamiento de la atención a la salud mental en la Comunidad Autónoma de Extremadura.

Decreto de 24 de mayo de 1943, sobre Dispensarios de Higiene mental.

Decreto del 7 de abril de 1851 del Boletín

Oficial de la Provincia de Badajoz. Artículo de

oficio. Circular Número 117

Decreto del 23 de enero de 1843 del Boletín Oficial de la Provincia de Badajoz. Artículo de Oficio. Circular Número 10

Delgado Bueno, S., \& González De Rivera y Revuelta, J. L. (1988). Aspectos Legales de los Internamientos Psiquiátricos. Psiquis, IX(88), 300-313.

Desviat, M.(1990). Epistemología y práctica psiquiátrica. Madrid: Ed. AEN.

Foucault, M. (1997). Historia de la locura en la época clásica. México: Fondo de Cultura Económica

Goffman,E.G.(1998).Internados. Ensayo sobre la situación social de los enfermos mentales. Buenos Aires: Amorrortu Editores.

González De Chaves, M.(1980). La transformación de la asistencia psiquiátrica.(Pp. 371-378). Madrid: Mayoría.

Kroll, J (1973). A reappraisal of psychiatry in the middle age. Archives of general psychiatry, 26, 276-283.

Leal Rubio, J.(1995). El manicomio. En Aguirre, A. , Rodríguez, A. Patios abiertos y patios cerrados.(pp. 53-69). Barcelona: Ed. Marcombo. 